\title{
Efficacy of massage on muscle soreness, perceived recovery, physiological restoration and physical performance in male bodybuilders
}

\begin{abstract}
It is believed that sport massage after intensive exercise might improve power and perceptual recovery in athletes. However, few studies have been done in this area. This study aimed to examine the effect of massage on the performance of bodybuilders. Thirty experienced male bodybuilders were randomly assigned to either a massage group $(n=15)$ or a control group $(n=15)$. Both groups performed five repetition sets at $75 \ddot{1} 77 \%$ of $1 \mathrm{RM}$ of knee extensor and flexor muscle groups. The massage group then received a 30-min massage after the exercise protocol while the control group maintained their normal passive recovery. Criteria under investigation included: plasma creatine kinase (CK) level, agility test, vertical jump test, isometric torque test, and perception of soreness. All variables were measured over 6 time periods: baseline, immediately after the DOMS inducing protocol, right after the massage, and 24, 48, and $72 \mathrm{~h}$ after the massage. Both groups showed significant $(\mathrm{P}<.001)$ decreases in jumping, agility performance, and isometric torque, but significant $(\mathrm{P}<.001)$ increases in $\mathrm{CK}$ and muscle soreness levels. The massage group in general demonstrated a better recovery rate. As such, a post-exercise massage session can improve the exercise performance and recovery rate in male bodybuilders after intensive exercise.
\end{abstract}

Keyword: Delayed onset; DOMS; Knee flexion; Resistance training; Therapy 